Har noni $c$ fi el $d$ cal cul at $i$ on by the combi nat $i$ on of $\mathrm{fi}$ i te el ement anal ysi s and harmic bal ance nethod

\begin{tabular}{|l|l|}
\hline 著者 & Yamada Sot oshi, Bessho Kazuo \\
\hline $\begin{array}{l}\text { j our nal or } \\
\text { publ i cat } \mathrm{i} \text { on } \mathrm{t} \text { i t I e }\end{array}$ & I EEE Tr ansact i ons on Nagget i cs \\
\hline vol une & 24 \\
\hline nunber & 6 \\
\hline page r ange & 2588 2590 \\
\hline year & 1988 11-01 \\
\hline URL & ht t p: //hdl . handl e. net /2297/48305 \\
\hline
\end{tabular}




\title{
HARMONIC FIELD CALCULATION BY THE COMBINATION OF FINITE ELEMENT ANALYSIS AND HARMONIC BALANCE METHOD
}

\author{
Sotoshi Yamada \\ Kazuo Bessho \\ Faculty of Technolog;, Kanazawa University \\ Kodatsuno 2-40-20, Kanazawa, Japan
}

Abstract - A new finite element analysis for the timeperiodic magnetic field calculation is described. An AC magnetic field calculation involving saturation characteristics requires many iterations and much memory region. The combination of FEM and harmonic balance method enables us to economically calculate harmonic magnetic, flux distributions at an $A C$ magnetization. The method does not need the calculation concerned with time and the the calculation algorithm is the same on the nonlinear $D C$ field FEM. The method is applied to the magnetic field analysis of a reactor and an electromagnet.

\section{Introduction}

There have been difficulties in the numerical calculation of time-periodic magnetic field incorporating saturation characteristics, because the treatment requires many iterations and much memory region. $[1,2]$

The harmonic balance method is of widest utility for obtaining the approximate periodic solution of a nonlinear differential equation. [3] Therefore, we propose the combination of FEM and the harmonic balance method and applied it to the harmonic magnetic field distribution problem with magnetic saturation. This method is called the harmonic balance finite element method (HBFEM).

\section{Formulation of HBEEM}

For simplicity, a magnetic field is assumed as two-dimensional in the $(x, y)$ plane and quasistationary. Therefore, the vector potential $A=(0,0, A)$ satisfics in the region of interest surrounded with some boundary conditions,

$$
\frac{\partial}{\partial x}\left(\nu \frac{\partial A}{\partial x}\right)+\frac{\partial}{\partial y}\left(\nu \frac{\partial A}{\partial y}\right)=-J_{0}+\sigma \frac{\partial A}{\partial t}
$$

where $v$ and $\sigma$ are magnetic reluctivity and conductivity.

Formulation is made by use of the Galerkin procedure and the weighting functions are the same as the shape functions $N_{i}(x, y)$. Its integral form is,

$$
\begin{aligned}
& \iint\left\{\frac{\partial N_{i}}{\partial x}\left(\nu \frac{\partial A}{\partial x}\right)+\frac{\partial N_{i}}{\partial y}\left(\nu \frac{\partial A}{\partial y}\right)\right\} d x d y- \\
& \iint\left(J_{0}-\sigma \frac{\partial A}{\partial t}\right) N_{i} d x d y
\end{aligned}
$$

We are only interested in the time-periodic solution (harmonic problem) when alternating current is applied. According to the harmonic balance method, all variables, i.e. vector potentials, flux densities and applied current, are approximated as harmonic solutions. For simplicity, the time-periodic solutions are given as the sum of the fundamental component and the third harmonic, that is,

$A^{i}=A_{1} s^{\prime} \sin \omega t+A_{1} c^{i} \cos \omega t+A_{3} s^{i} \sin 3 \omega t+A_{3 c^{i}} \cos 3 \omega t$

$B_{x}=B_{x 1}, \sin \omega t+B_{x 1} \cos \omega t+B_{x}, \sin 3 \omega t+B_{x 3} \cos 3 \omega t$

$B_{y}=B_{y 1 s} \sin \omega t+B_{y 1 c} \cos \omega t+B_{y 3} \sin 3 \omega t+B_{y 3} \cos 3 \omega t$

$J_{0}=J_{1} \sin \omega t+J_{1} \cos \omega t+J_{3} \sin 3 \omega t+J_{3} \cos 3 \omega t$

where $\omega$ is the fundamental angular frequency.

The magnetizing characteristics of a core can be expressed as a power series. The B-ll curve is approximated as a third order power series, that is,

$$
\mathrm{H}=\alpha \mathrm{B}+\beta \mathrm{B}^{3}
$$

where hysteresis characteristic is neglected. The reluctivity is written as,

$$
\nu=\alpha+\beta B^{2}
$$

where $B=\left(B x^{2}+B_{y}{ }^{2}\right)^{1 / 2}$. We substitute Eqs. (3) and (5) into (2) and equate the coefficients of $\sin (n \omega t)$ and $\cos (n \omega t)(n=1,3)$ on both sides. As a result, the matrix for one element is expressed as,

$\frac{1}{A \Delta}\left[\begin{array}{lll}\left(b_{1} b_{1}+c_{1} c_{1}\right) D & \left(b_{1} b_{2}+c_{1} c_{2}\right) D & \left(b_{1} b_{3}+c_{1} c_{3}\right) D \\ \left(b_{2} b_{1}+c_{2} c_{1}\right) D & \left(b_{2} b_{2}+c_{2} c_{2}\right) D & \left(b_{2} b_{3}+c_{2} c_{3}\right) D \\ \left(b_{3} b_{1}+c_{3} c_{1}\right) D & \left(b_{3} b_{2}+c_{3} c_{2}\right) D & \left(b_{3} b_{3}+c_{3} c_{3}\right) D\end{array}\right]\{A\}$

$$
+\frac{\sigma \omega \Delta}{12}\left[\begin{array}{ccc}
2 N & N & N \\
N & 2 N & N \\
N & N & 2 N
\end{array}\right]\{A\}-\{K\}
$$

where

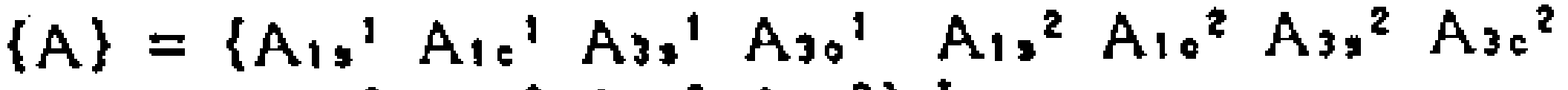

$$
\begin{aligned}
& \left.A_{1} 0^{3} A_{10} A_{3} 3^{3} A_{30}\right\}^{3} \\
& \{\mathrm{~K}\}=\Delta / 3\left\{\mathrm{~J}_{13} \mathrm{~J}_{10} \mathrm{~J}_{35} \mathrm{~J}_{30} \mathrm{~J}_{13} \mathrm{~J}_{10} \mathrm{~J}_{33} \mathrm{~J}_{3 \mathrm{c}}\right. \\
& \left.\mathrm{J}_{1,}, \mathrm{~J}_{1 \mathrm{C}} \mathrm{J}_{33} \mathrm{~J}_{3 \mathrm{e}}\right\}^{\prime} \\
& b_{i}=y_{j}-y_{k}, c_{i}=x_{k}-x_{j}, \Delta \text { : cross section, }\left(x_{i}, y_{i}\right) \text { : node }
\end{aligned}
$$

The block matrices $D\left(4^{*} 4\right)$. and $N\left(4^{*} 4\right)$ are given as Eqs.A(1) and $A(2)$ in Appendix (A). The coefficients of the matrix $D$ are determined by both parameters of $B-H$ curve and the flux densities of each harmonic. Compared with the conventional FEM, it acts as a reluctivity and is called the reluctivity matrix. The matrix $N$ is a constant concerned with harmonic orders and is called the harnonic matrix.

The matrix equation for the entire region is obtained by the same procedure as the conventional FE' and is also solved by the same iteration procedure as a nonlinear static field.

As magnetic saturation becomes stronger, it is necessary to approximate characteristic by higher order power series. The $\mathrm{B}-\mathrm{H}$ curve is expressed as a fifth order power series as,

$$
\mathrm{H}=\alpha \mathrm{B}+\beta \mathrm{B}^{3}+\gamma \mathrm{B}^{5}
$$

Then, the reluctivity is given as..

$$
\nu^{\prime}=\alpha+\beta \mathrm{B}^{2}+\gamma \mathrm{B}^{\prime}
$$

The matrix for each element is the same as Eq.(6). Only the block matrix $D$ is changed and is given in Appendix (B). The matrix $N$ is the same in Appendix (A).

The above method does not require the calculation concerned with time and can solve the time-periodic field distributions of $A C$ machines with nonlinear characteristics. But the system matrix is four times bigger than the number of vertices when the fundamental and third harmonic components are considered.

\section{Verification and Applications of the Method}

Reactor

In order to verify HBFEM, the procedure is applied to a reactor with a saturated core as shown in Fig.l(a). The domain for calculation is subdivided as shown in Fig.l(b). Eddy currents in the core are neglected and the B-H curve of the core is given as a third order power series in Fig.2. When the fundamental and third harmonic components of the applied current density are given, each component of flux density in the middle leg is obtained in Fig.3. When the third harmonic of flux density attains the minimum, the magnetic flux density is sinusoidal. 
The waveforms of flux density in the middle leg are drawn in Fig.4(a) and (b) when each of the applied current and flux density is sinusoidal. In this problem, it is possible to calculate the flux distribution by using the ordinary technique of nonlinear static FEM as the current density is given at a particular instant. [4] The points illustrated as circles indicate the results. The agreement of the two results verifies the procedure of harmonic balance FEM.

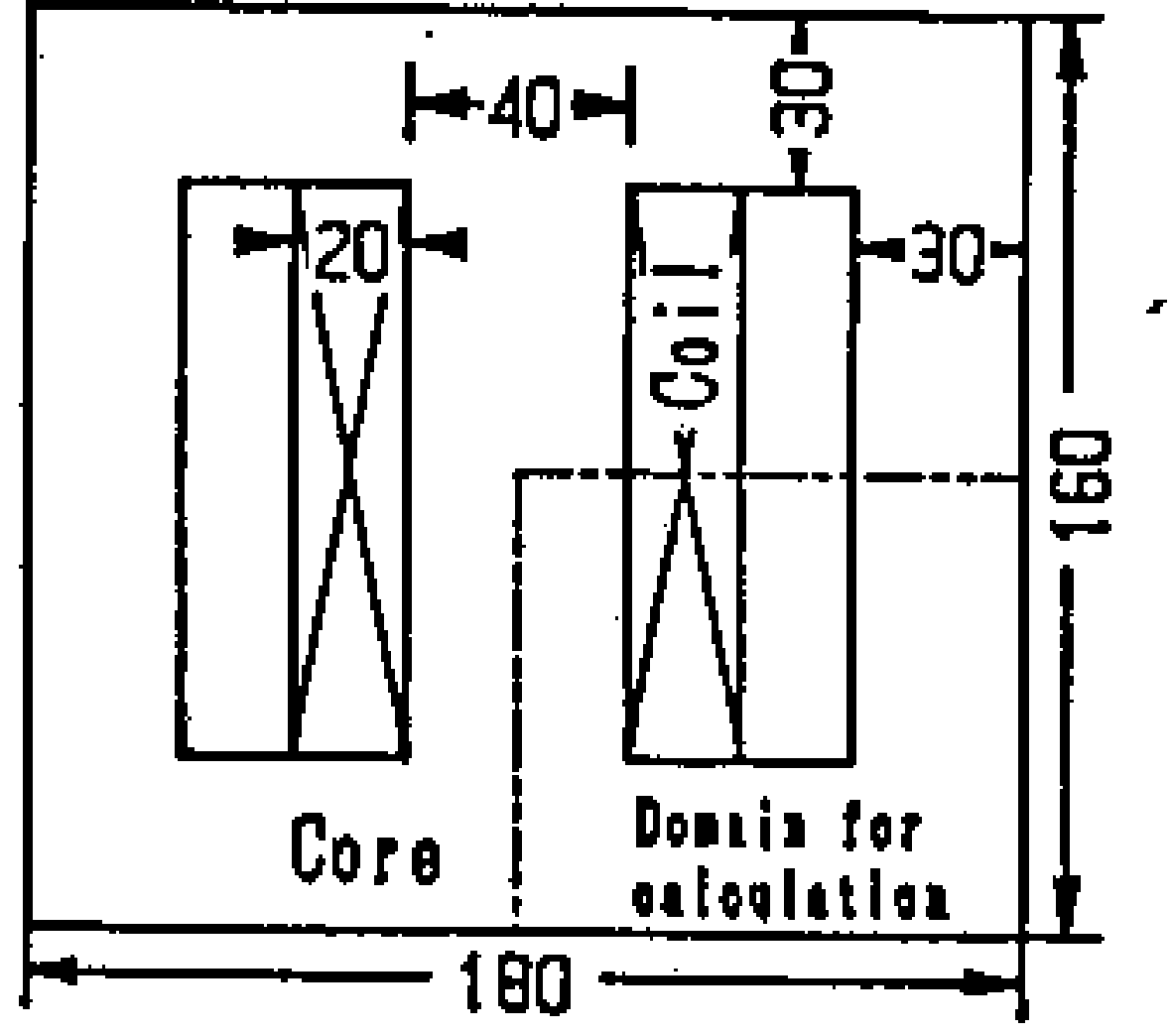

(a) Reactor

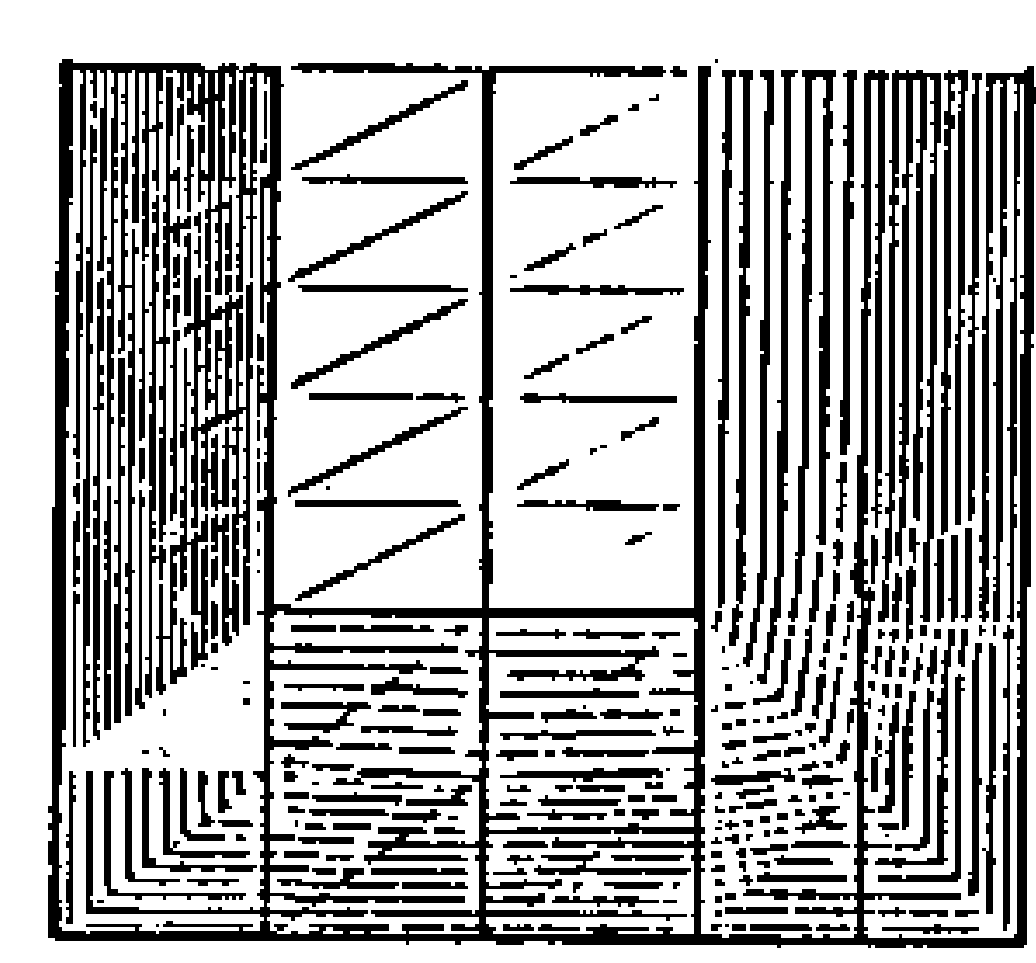

(b) Mesh and distribution
Fig.1 Reactor with saturated core

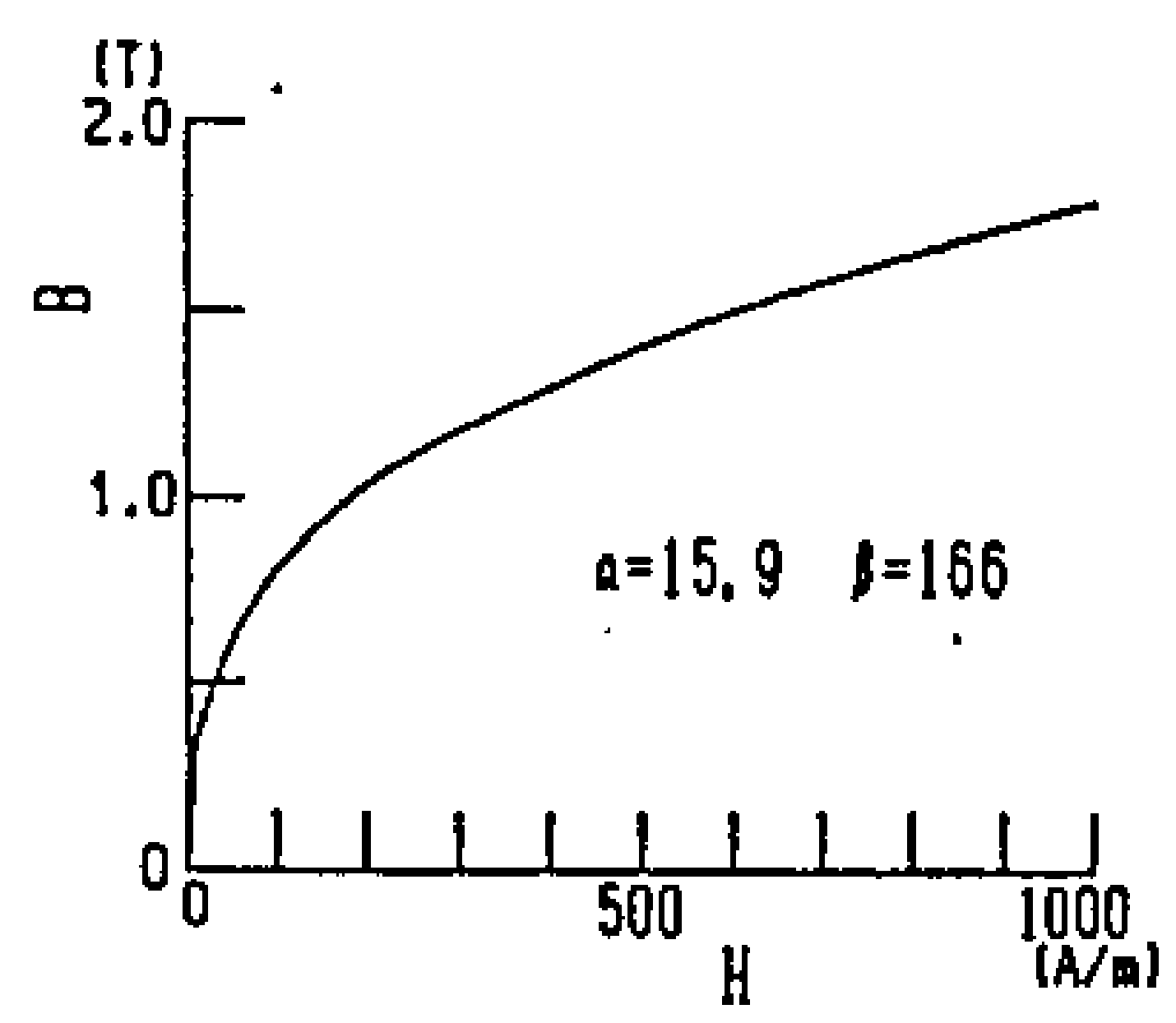

Fig.2 B-H curve of saturated core

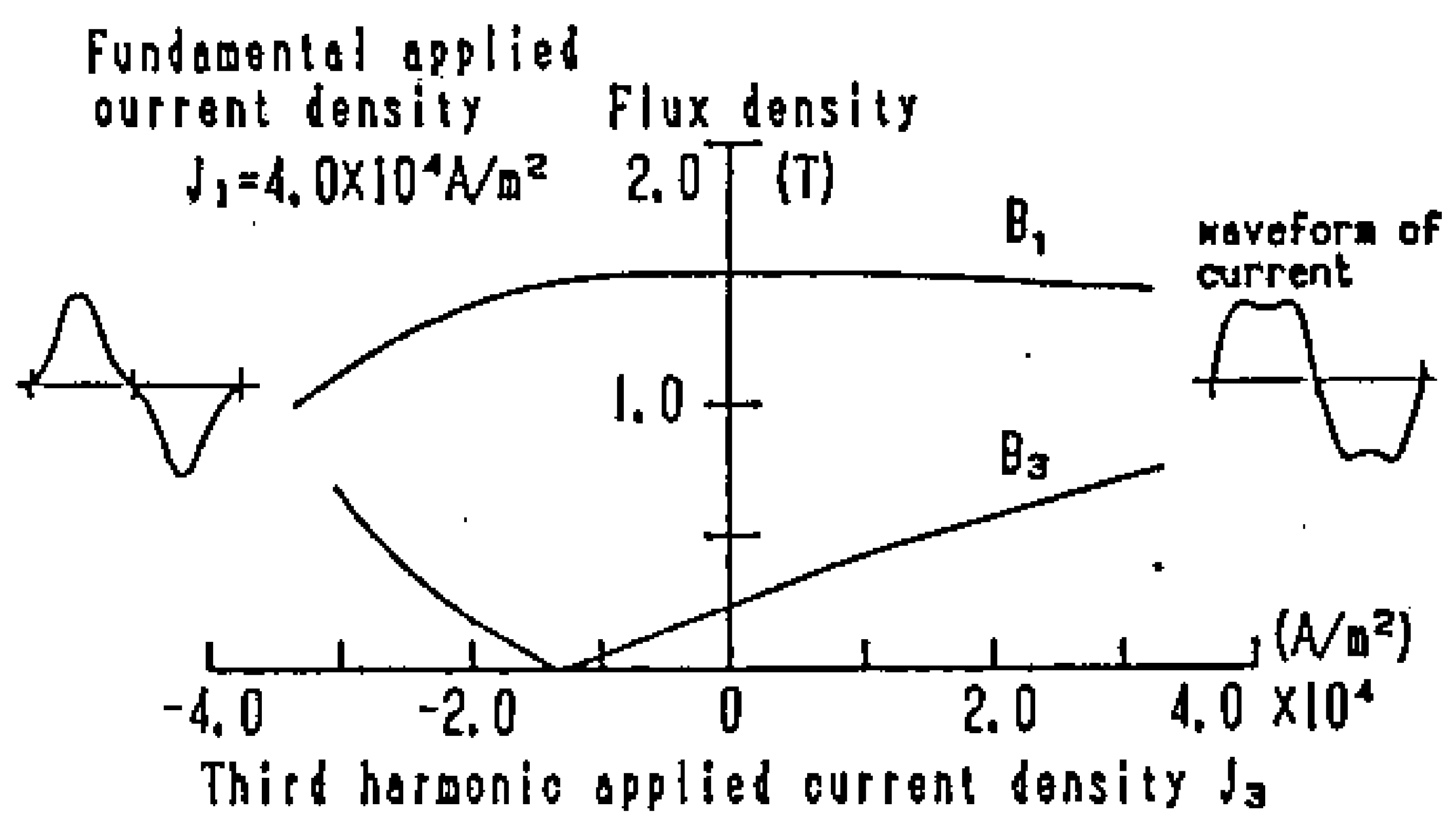

Fig. 3 Magnetic flux density in the middle leg when the fundamental and third harmonic currents are applied.

Magnet with Shading Coil

The IIBFEM is applied to the field calculation of the electromagnet in Fig.5. The middle leg has an airgap and shading coils. In this case, the magnetizing characteristics of an iron core are expressed as a fifth order power harmonic. The parameters for calculation are given as,

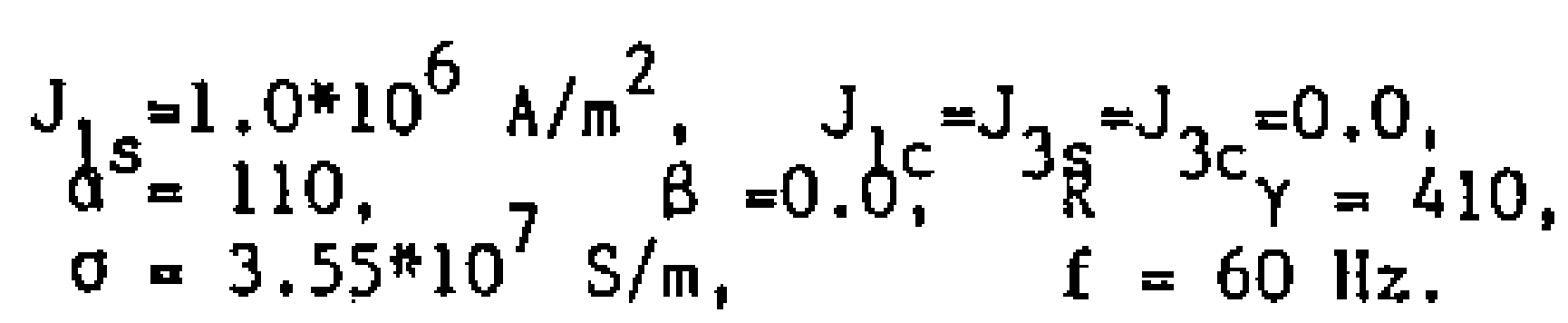

The distributions of each component in Fig. 6 are drawn at a particular phase. The HBFEM directly gives the component of each harmonic.
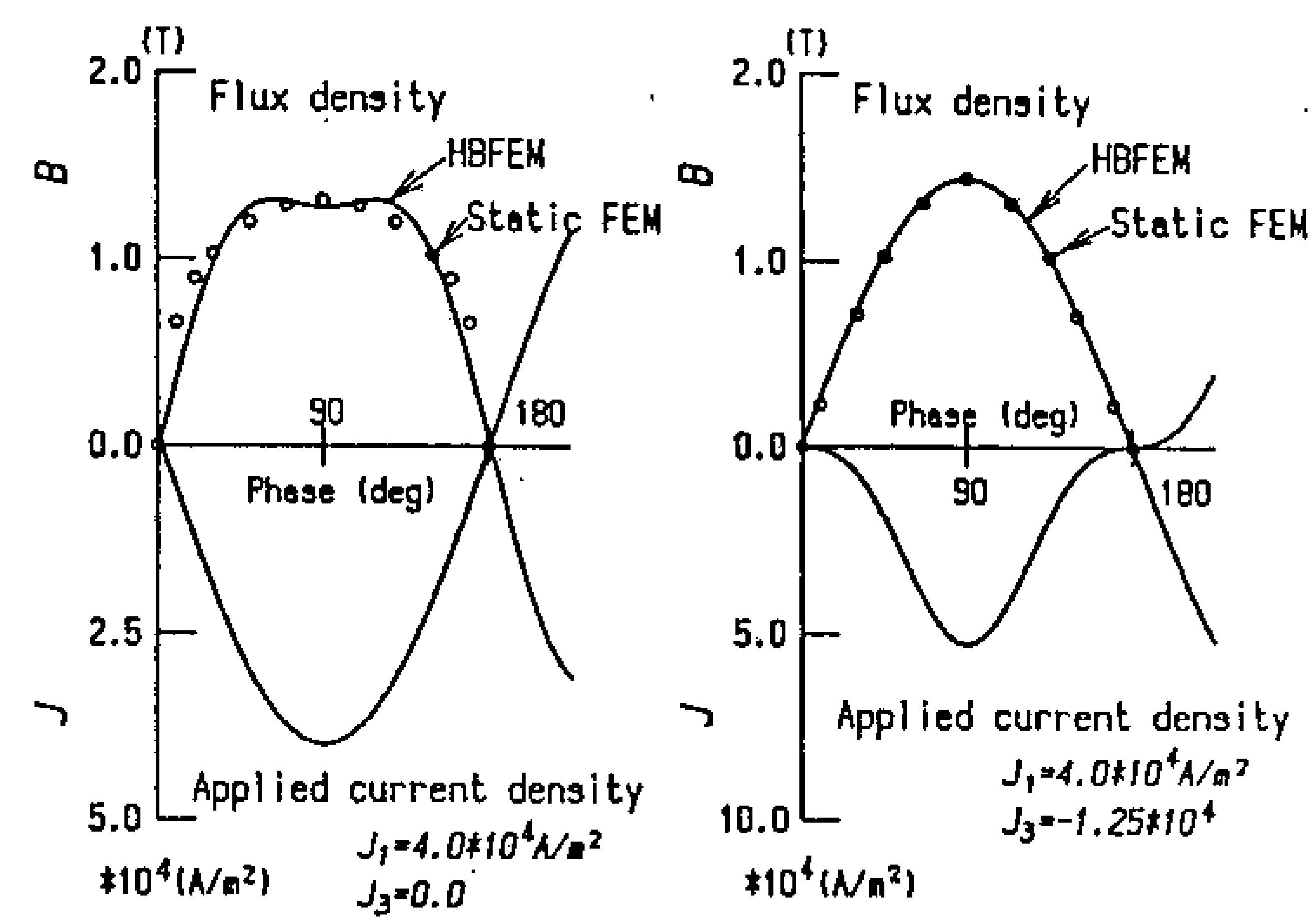

(a) The applied current is sinusoidal

(b) The flux density in the middle leg is sinusoidal

Fig. 4 Comparisons with results calculated by HBFEM and nonlinear static FEM

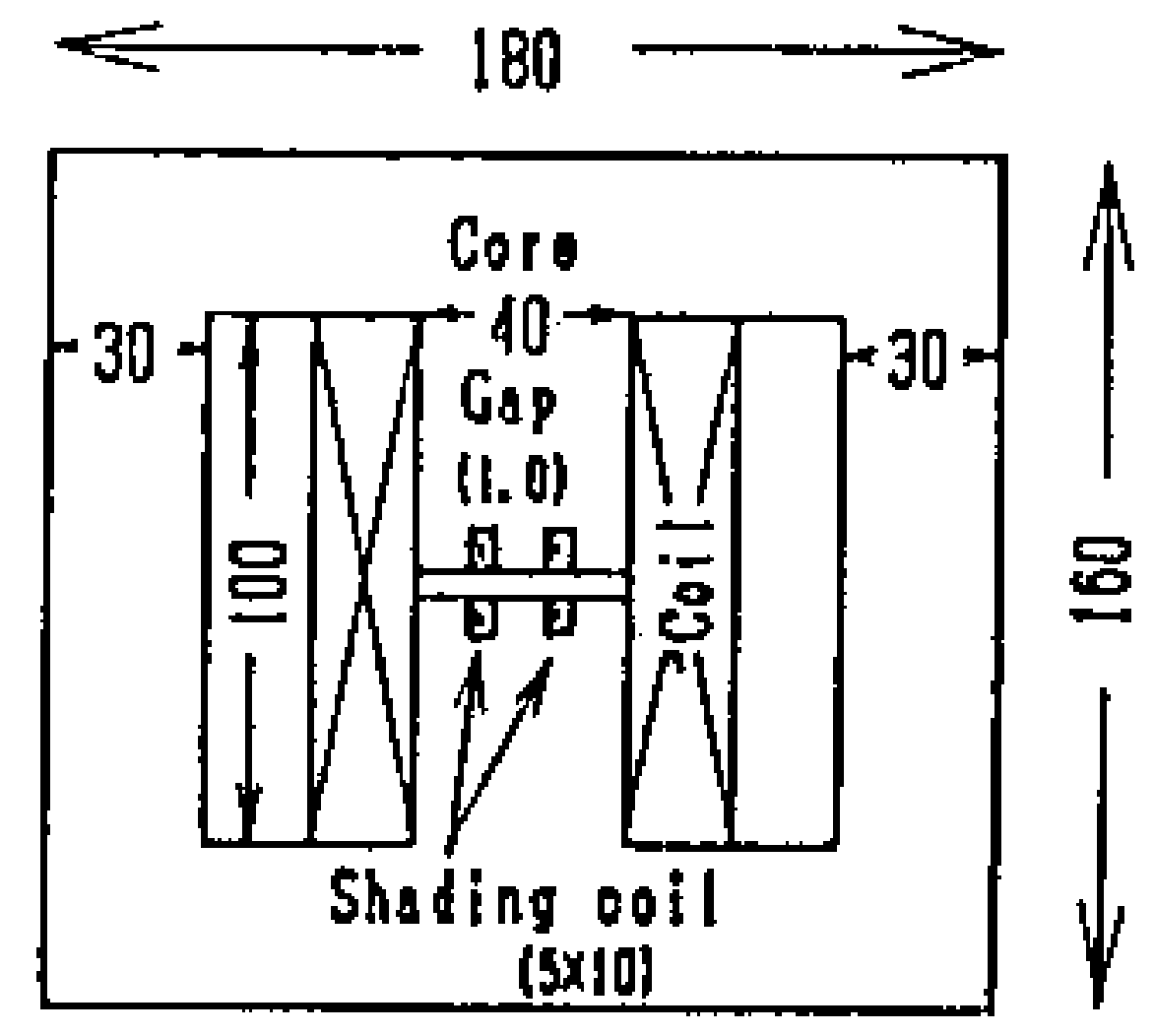

Fig.5 Electromagnet with shading coils
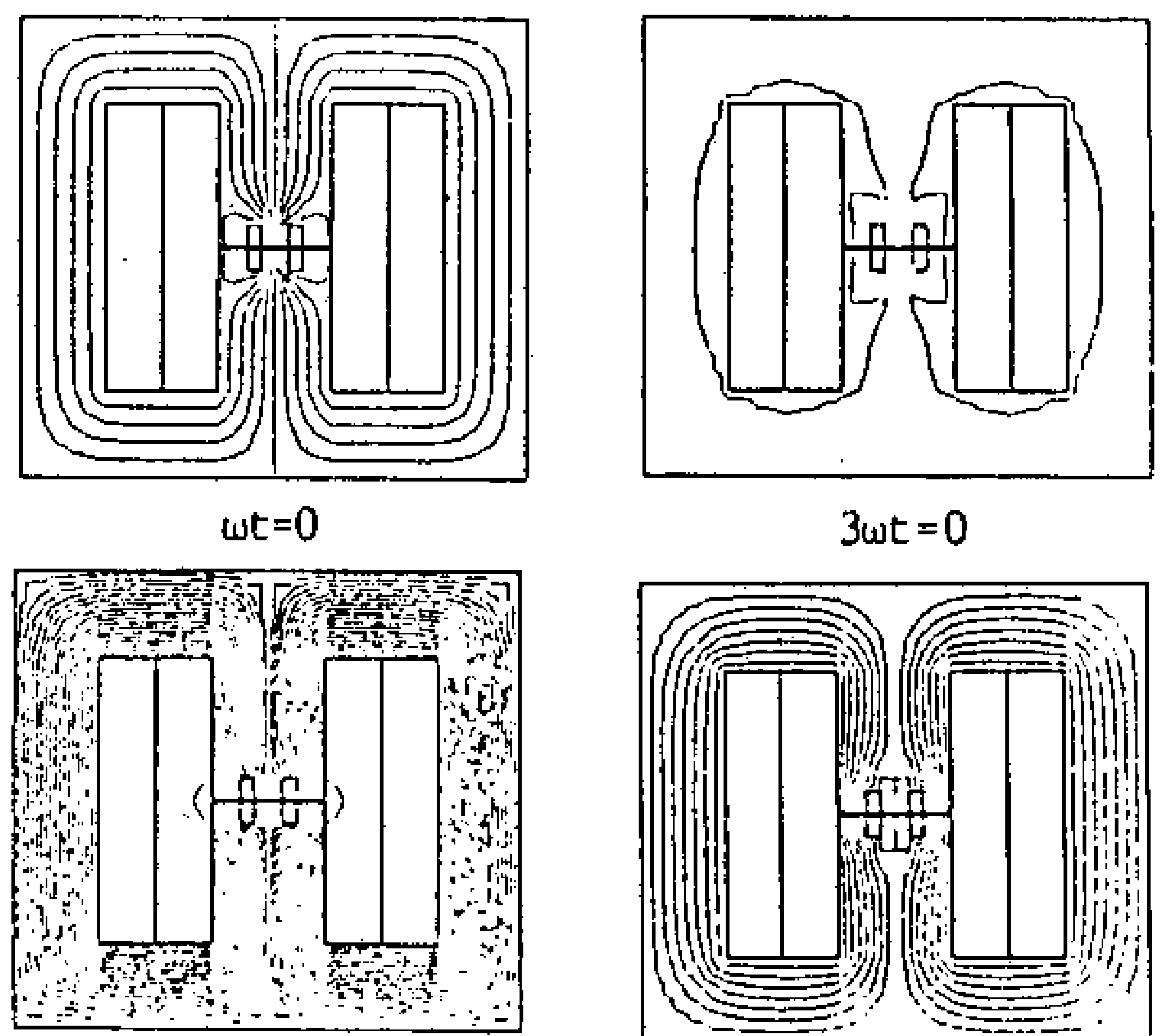

$\omega t=90 \mathrm{deg}$

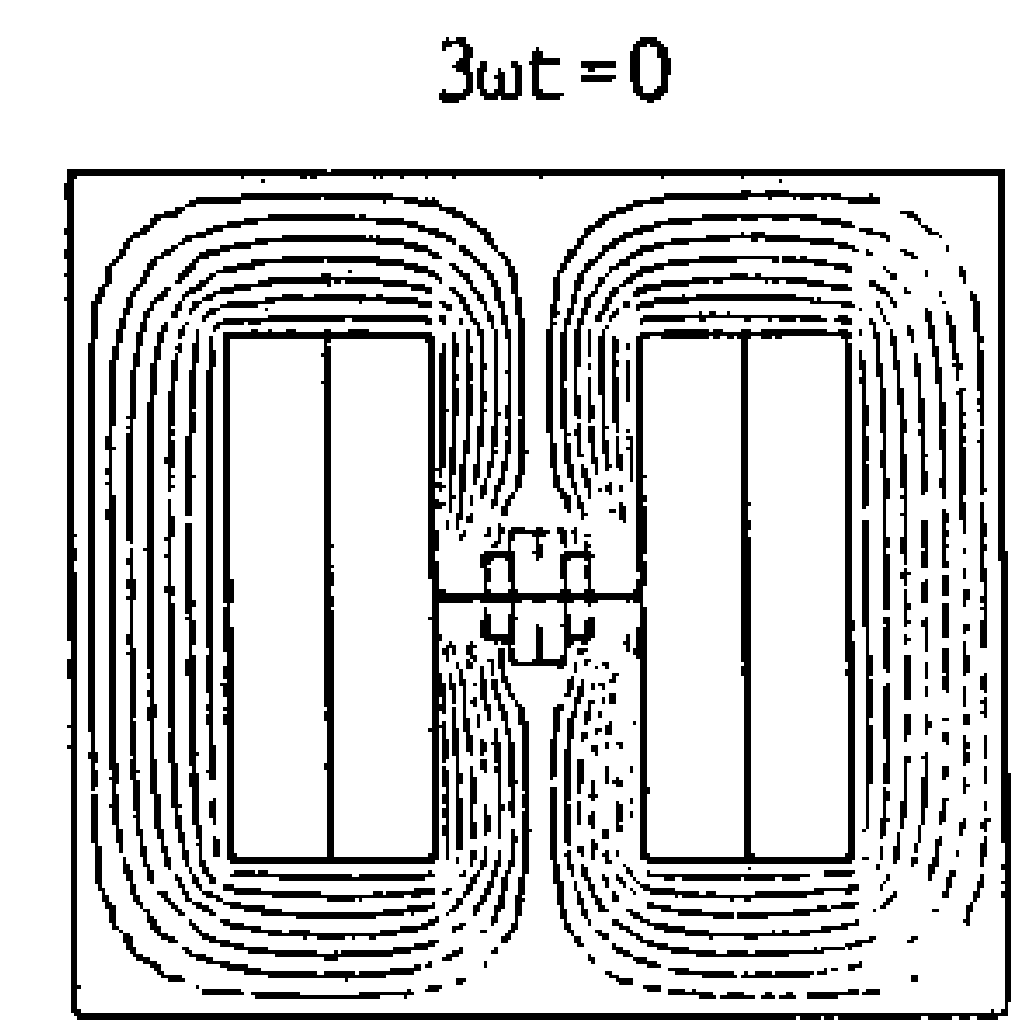

$3 \omega t=90$ deg

(a) Fundamental component (b) Third harmonic component Fig.6 Flux distribution

\section{Conclusions}

$A$ new FEM technique for time-periodic field distribution with magnetic saturation characteristics is proposed. The procedure of the calculation is the same as the static nonlinear FEM and does not include the calculation of the time component. 


\section{References}

[1] M.V.K. Chari, et al : Finite Element in Electrical and Magnetic Field Problems, John Wiley \& Sons, $1980, \mathrm{Ch}, 8$.

(2] T.Hara, T.Naito, J.Umoto ; "Time-periodic Finite Element Method for Nonlinear Diffusion Equation", IEEE Trans. Magnetics, Vol.MAG-21,No.6, pp22612264,1985 .

[3] C.Hayashi : Nonlinear Oscillations in Physical System, McGraw Hill, 1964, Ch. 1 \& 5 .

[4] P.Silvester, M.V.X.Chari :"Finite Element Solution of Saturable Magnetic Field Problem", IEEE Trans. PAS, Vol.PAS-89, No.7, ppl642-1649, 1970.

\section{Appendix A}

The block matrices $D$ and $N$ are given as,

$D=\left[\begin{array}{cccc}\alpha+\beta\left(B_{0}-B_{2 c} / 2\right) & \beta B_{2 s} / 2 & \beta\left(B_{2 c}-B_{c c}\right) / 2 & \beta\left(B_{4,}-B_{2 s}\right) / 2 \\ \alpha+\beta\left(B_{0}+B_{2 c} / 2\right) & \beta\left(B_{2 s}+B_{a},\right) / 2 & \beta\left(B_{2 c}+B_{4 c}\right) / 2 \\ \text { symmetry } & \alpha+\beta\left(B_{0}-B_{b c} / 2\right) & \beta B_{b s} / 2 \\ & \alpha+\beta\left(B_{0}+B_{b c} / 2\right)\end{array}\right]$

$N=\left[\begin{array}{cccc}0 & -1 & 0 & 0 \\ +1 & 0 & 0 & 0 \\ 0 & 0 & 0 & -3 \\ 0 & 0 & +3 & 0\end{array}\right]$

The coefficients in the matrix $D$ are expressed "as a function of Flux densities and described as,

$B_{B}=\left(B_{x 1} s^{2}+B_{x} c^{2}+B_{x} s^{2}+B_{x} c^{2}\right) / 2+\left(B_{y 1} s^{2}+B_{y} c c^{2}+B_{y 3} s^{2}+B_{y} c^{2}\right) / 2$ $B_{2},=B_{x 1}, B_{x 1 c}+B_{x 1} B_{x 3},-B_{x 1}, B_{x 3}+B_{y 1}, B_{y 1 c}+B_{y 1}<B_{y 3},-B_{y 1}, B_{y 3}$ $B_{2 c}=\left(B_{x} \times c^{2}-B_{x 1} s^{2}\right) / 2+B_{x 1,}, B_{x 3},+B_{x 1 c} B_{x 3 c}$

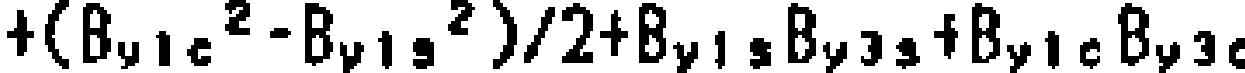

$B_{4}=B_{x 1}, B_{x 3}+B_{x 1 c} B_{x 3},+B_{y 1}, B_{y}{ }_{3 c}+B_{y 1}+B_{y} s$,

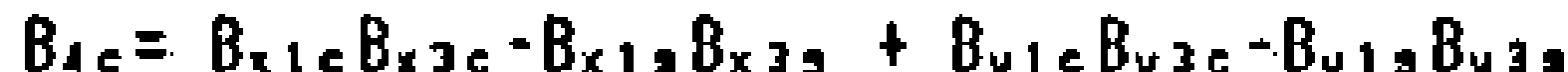

$B_{6 s}=B_{x 3}, B_{x 3 c}+B_{y 3} B_{y 3 c}$

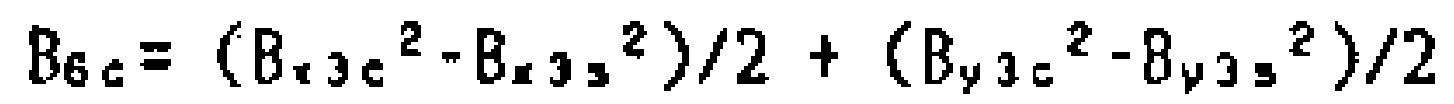

\section{Appendix B}

The reluctivity matrix $D$ is expressed as,

$$
D=D x+D x y+D y
$$

where $D x, D x y$, and Dy are given as
$B_{x y}=\left(B_{x}+s^{2}+B_{x}+c^{2}+B_{x} g^{2}+B_{x 3 c^{2}}\right) / 2$

$B_{\times 2}=B_{x 1}, B_{x 1 C}+B_{x 1} \in B_{x 3},-B_{x 1}, B_{x} 30$

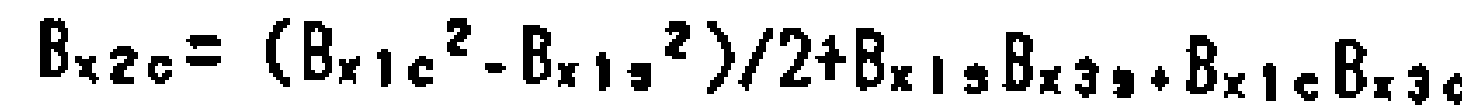

$B_{x 43}=B_{x 1}, B_{x 3}+B_{x}+B_{x 3}$,

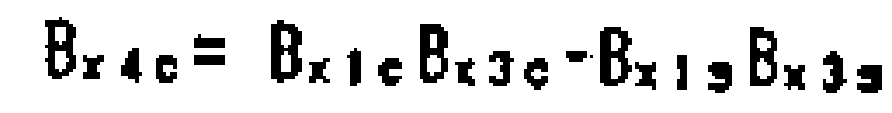

$B_{\times 8}=B_{2}=3 B_{3} B_{0}$

$B_{x B c}=\left(B \times 30^{2}-B \times 3 s^{2}\right) / 2$

$B_{y b}=\left(B_{y 1} s^{2}+B_{y} 1 c^{2}+B_{y 3} z^{2}+B_{y 3} c^{2}\right) / 2$

$B_{y 2}=B_{y 1}, B_{y 1 c}+B_{y 1}, B_{y 3},-B_{y 1}, B_{y j}$

$B_{y 2 c}=\left(B_{y} 1 c^{2}-B_{y} 1^{2}\right) / 2+B_{y 1}, B_{y 3} y+B_{y 1} B_{y 3 c}$

$B_{y 4}=B_{y 1}, B_{y 3 c}+B_{y 1}+B_{y 3}$ s

$B_{y 4 c}=B_{y 1} c B_{y 3 c}-B_{y 1}+B_{y 3}$,

$B_{48}=B_{y 3}, B_{y 3 c}$

$B_{y b c}=\left(B_{y 3 c} c^{2}-B_{y j} g^{2}\right) / 2$

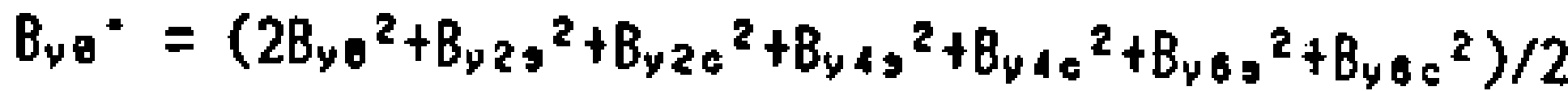

$B_{y 2} y^{\circ}=2 B_{y 0} B_{y 2},-B_{y 2}, B_{y<c}+B_{y 2} e B_{y 4},-B_{y 4}, B_{y 6 c}+B_{y 4 c} B_{y}$,

$B_{y 2 c^{\circ}}=2 B_{y \theta} B_{y z c}+B_{y z}, B_{y d}, B_{y z} 0 B_{y<c}+B_{y 4}, B_{y b},+B_{y 4 c} B_{y b c}$

$B_{y 4 s^{\circ}}=2 B_{y} B_{y 4 s}+B_{y z}, B_{y 2 c}-B_{y z}, B_{y 6 c}+B_{y 2} B_{y b}$,

$B_{y 4 c}=\left(B_{y} c^{2}{ }^{2}-B_{y 2} s^{2}\right) / 2+2 B_{y} B_{y<c}+B_{y 2} B_{y b},+B_{y z} B_{y 6 c}$

$B_{y 8 s}=2 B_{y z} B_{y 6}, B_{y 2}, B_{y} B_{c}+B_{y 2} B_{y y s}$

$B_{y b c}=2 B_{y a} B_{y b c}-B_{y z} g B_{y 4}+B_{y z e} B_{y d c}$

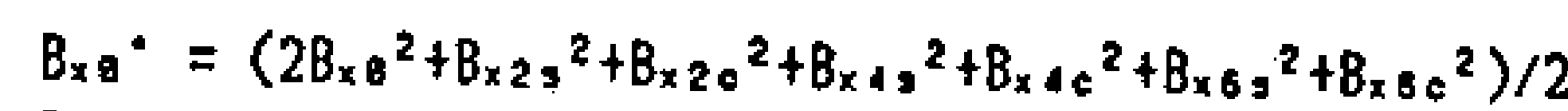

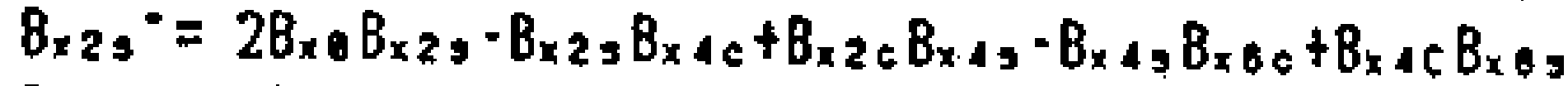

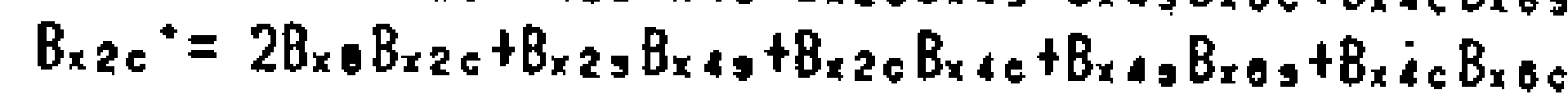

$B_{x 4}{ }^{\circ}=2 B_{x 0} B_{x 4},+B_{x 2}, B_{x 2} c^{-}-B_{x 2}, B_{x 6 c}+B_{x 2} B_{x} B_{x s}$

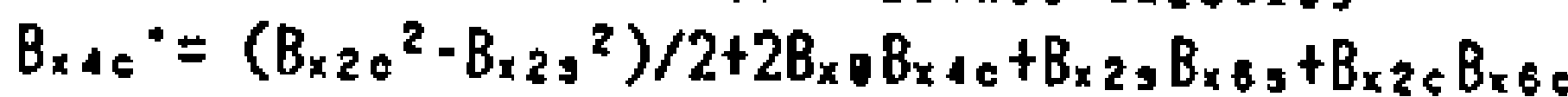

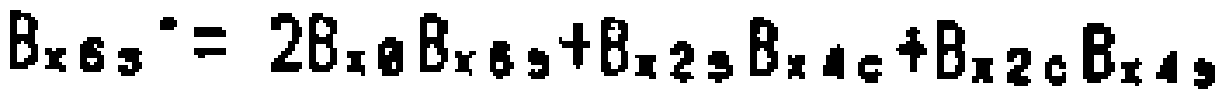

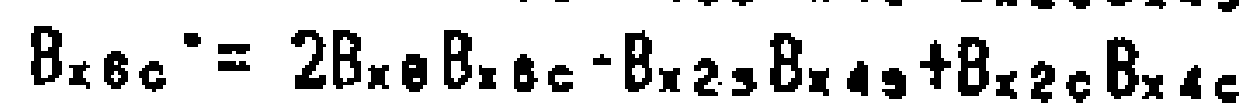

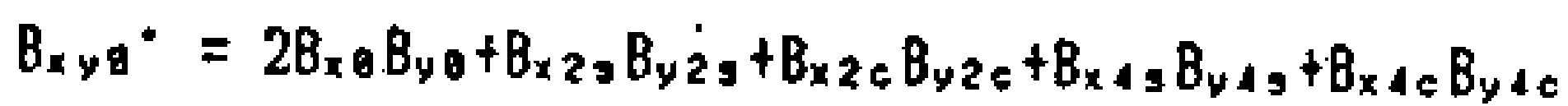

$+B_{x} 8 B_{y} B_{0}+B_{x 6}=B_{y 6 c}$

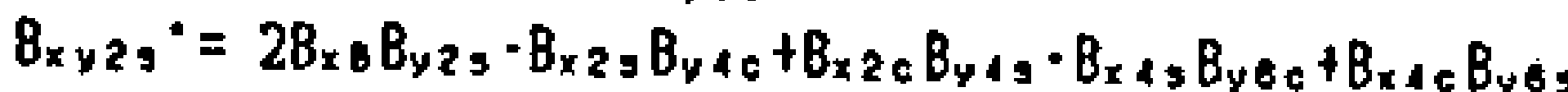

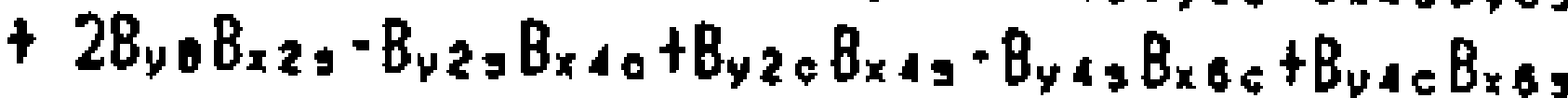

$B_{x y z c}=2 B_{x \theta} B_{y z c}+B_{x 2 s} B_{y+g}+B_{x 2 c} B_{y 4 c}+B_{x 4} B_{y e}+B_{x+c} B_{y 6 c}$

$+2 B_{y \theta} B_{x z c}+B_{y z} s B_{x 4} s+B_{y 2 c} B_{x 4 c}+B_{y 4}, B_{x b}+B_{y 4 c} B_{x b c}$

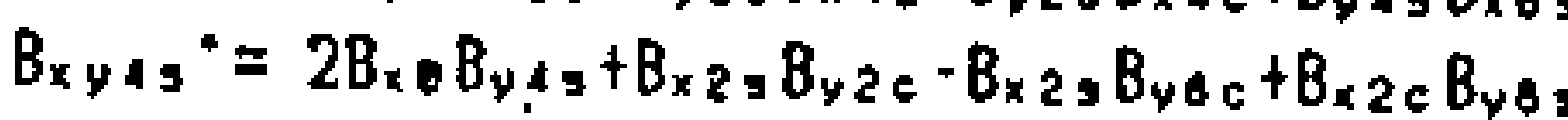

$+2 B_{y, 0} B_{x} 43+B_{y 2}, B_{x 20}-B_{y 2}, B_{x 8}+B_{y 2}=B_{x 3}$

$B_{x y 4 c}=\left(B_{x 20} B_{y 2 c}-B_{x 2}, B_{y 2} s\right) / 2+2 B_{x}, B_{y 4 c}+B_{x 2}, B_{y s}+B_{x 2}=B_{y 8 c}$

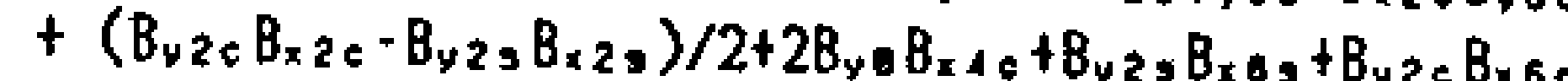

$B_{x y 8} y^{\circ}=2 B_{x 8} B_{y 6},+B_{x 2} 3 B_{y 4 c}+B_{x 2 c} B_{y 43}$

$+2 B_{y \theta} B_{x} b s+B_{y 2}, B_{x 4 c}+B_{y z} a B_{x} 4$,

$B_{x y c_{c}}=2 B_{x} B B_{y 8 c}-B_{x 2}, B_{y 4}, B_{x 2}+B_{y 4 c}$

$+2 B_{y 8} B_{x \in c}-B_{y 2}, B_{x 4},+B_{y 2}<B_{x 4 c}$

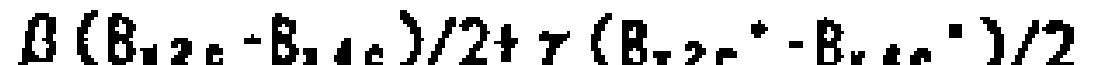

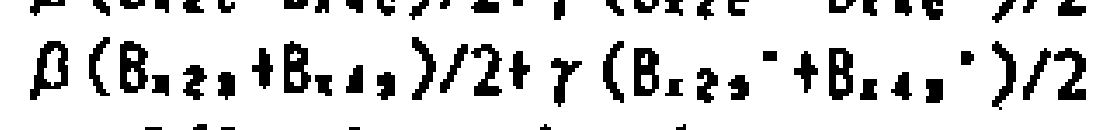

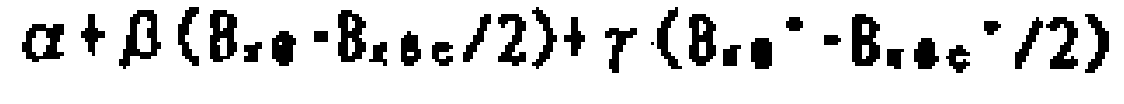

$B\left(B_{14},-B_{x 2},\right) / 2+\gamma\left(B_{14},-B_{1}, 1^{\circ}\right) / 2$ $\beta\left(B_{i 2 e}+B_{2+C}\right) / 2+\gamma\left(B_{12} e^{*}+B_{4}+\varepsilon^{\circ}\right) / 2$ $B_{1}, 6,2+\gamma B_{26} 3^{\circ} / 2$

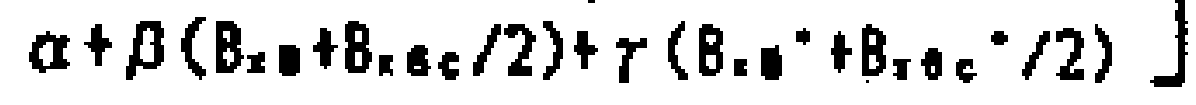

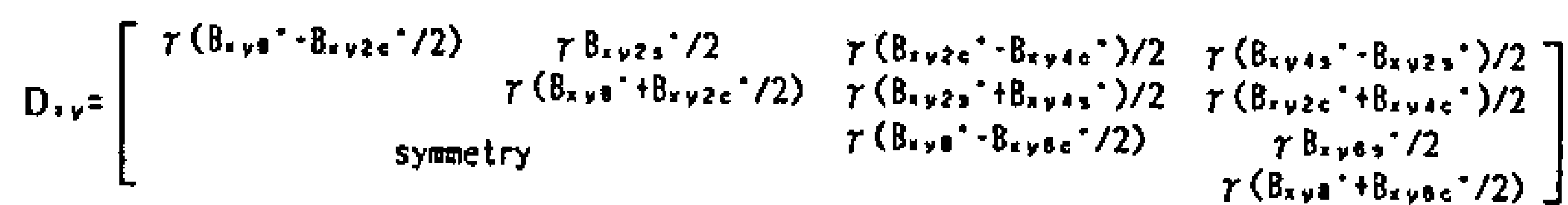

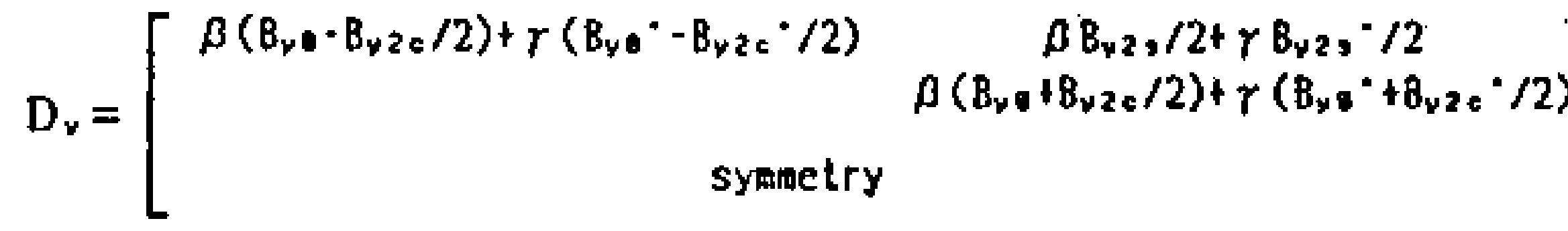

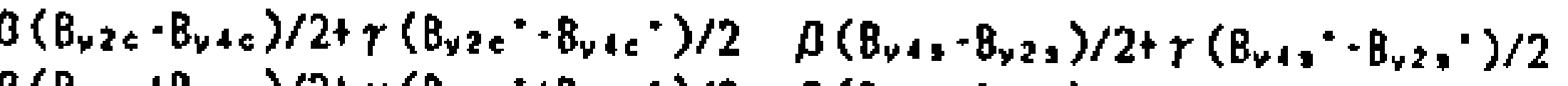

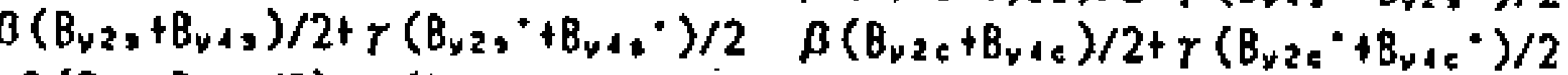

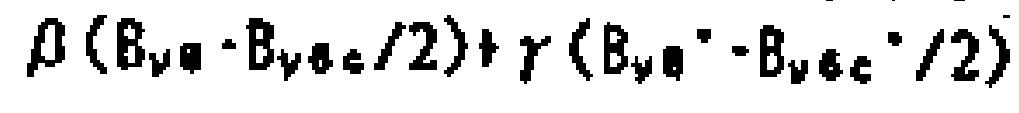

$\beta B_{y 8}, / 2+\gamma B_{y s}, \angle / 2$ $\beta\left(B_{y}+B_{y s} / 2\right)+T\left(B_{y 0^{*}}+B_{y b c} / 2\right)$

The coefficients in Eqs, $B(2), B(3)$ and $B(4)$ are 